\title{
Phonon properties and photo-thermal oxidation of micromechanically exfoliated antimonene nanosheets
}

Michael Fickert, Mhamed Assebban, Josep Canet-Ferrer and Gonzalo Abellán

\begin{abstract}
Two-dimensional (2D) sheets of antimonene have attracted increasing attention due to their unique physical and chemical properties prompting potential for diverse applications. We present a facile method to prepare high-quality antimonene nanosheets (ANSs) by micromechanical exfoliation on $\mathrm{SiO}_{2} / \mathrm{Si}$ substrate. The temperature- and laser power-dependent Raman studies of exfoliated ANSs are reported and analyzed. It was found that both the out-of-plane $A_{1 g}$ and the in-plane $E_{g}$ modes red-shift linearly with increase in temperature, pointing towards anharmonic vibrations of the lattice. The thermal response of the ANSs on a $\mathrm{SiO}_{2} / \mathrm{Si}$ surface is also described using numerical simulation of the heat transfer to study their laser-induced oxidation mechanisms. These results offer a deeper understanding of the phonon properties and oxidation susceptibility of $2 \mathrm{D}$ antimonene paving the way for the development of antimonene-based technologies, such as electronic devices or photothermal cancer therapy.
\end{abstract}

\section{Introduction}

The successful isolation of graphene in 2004 has triggered tremendous research interest in atomically thin two-dimensional (2D) crystals [1], including boron nitride (h-BN) [2] and transition metal dichalcogenides (TMDCs) [3], owing to their unique electronic, optical and mechanical properties compared with their bulk counterparts [4, 5]. Recently, monolayer semiconductors of group-15 (P, As, Sb, Bi), also known as 2D pnictogens are attracting increasing attention due to their extraordinary properties, like high carrier mobility [] ], layer-dependent tunable bandgaps [7] , and superior catalytic activity [무, $\underline{9}$, which makes them appealing materials for diverse applications in opto-electronic devices and beyond $[10,11]$. Among them, antimonene is of particular interest and was proposed as a promising 2D semiconductor with a band gap of $2.28 \mathrm{eV}$ [12] exhibiting a very high stability under ambient conditions and room temperature [13], as a clear advantage with respect to its homologue black phosphorus that suffers from oxidative degradation under similar conditions $[14,15]$. In addition, it was observed that antimonene undergoes indirect-direct band-gap transition [16], semiconductor-metal transition [17], and even topological phase transition under tensile strain [18] and is therefore foreseen as an 
excellent candidate for future quantum devices [19]. Bulk hexagonal antimony ( $\beta$-phase) is the most stable antimony allotrope at ambient conditions. Similar to silicene, $\beta$ antimony has a layered structure that consists of vertical stacking of a non-planar two atoms-thick individual layers held together by van der Waal interactions [20]. In a monolayer antimonene, each $\mathrm{Sb}$ atom is covalently bonded to other three $\mathrm{Sb}$ atoms forming a buckled washboard-like structure [15].

Ultrathin antimonene layers have been experimentally prepared by several techniques such as mechanical exfoliation [21], liquid-phase exfoliation [22-24], chemical vapor deposition (CVD), van der Waals epitaxial growth [25-27], and colloidal synthesis [28]. The synthesis of antimonene via mechanical exfoliation offers isolated samples with high quality and purity, which make it more feasible for researchers to investigate the fundamental properties of the material. However, up to now, there is only one work reporting the micromechanical isolation of antimonene [21], despite the simplicity of this method. Therefore, it is urgently needed to develop an effective exfoliation procedure to obtain high-quality antimonene for enabling fundamental research as well as for device fabrication.

Furthermore, in the view of antimonene as a possible material for nanodevices, it is imperative to understand its phonon and thermal properties. Actually, it is of technological importance to know the dynamics of phonons induced by self-heating of the device, which will have influence on the final performance. In this sense, temperature and power-dependent Raman spectroscopy can be used for noncontact and reliable demonstration of the anharmonic properties at the nanoscale [29-31]. This method has been widely used for the investigation of the anharmonic phonon and thermal properties of a wide range of 2D materials such as, graphene [32], black phosphorus [33] and TMDCs [34]. However, such fundamental understanding of phonon and thermal properties in the case of $2 \mathrm{D}$ antimonene remains unexplored.

Here, we introduce an adapted micromechanical method to easily isolate antimonene nanosheets (ANSs) down to the few-layer regime. The fabricated ANSs have been comprehensively characterized by optical microscopy, scanning electron microscopy (SEM), energy dispersive x-ray analysis (EDX), atomic force microscopy (AFM), and Raman spectroscopy. In order to gain a deeper insight into their thermal properties, temperature-dependent Raman spectroscopy was performed and corroborated with numerical simulations on the heat transfer. We found that increasing temperature the frequency of the Raman-active modes of antimonene red-shift linearly mediated by phonon-phonon interactions as explained on the basis of the anharmonic vibrations of the lattice. Furthermore, the thermal and photothermal oxidation of ANSs was 
demonstrated and analyzed. Our work introduces a reliable method to produce highquality ANSs and shed light on their thermal properties and oxidation susceptibility. These findings pave the way for a deeper understanding of phonon properties and oxidation susceptibility of 2D antimonene, which will contribute to the future development of efficient antimonene-based technologies with applications in opto-electronics and beyond.

\section{Results and discussion}

$\beta$-antimony, which crystallizes in the rhombohedral system forming a buckled honeycomb lattice, is the most stable allotrope of antimony. It is characterized by a layered structure in which the in-plane bonds are stronger than the van der Waals interlayer interaction. This difference between the strength of the in- and out-of-plane bonding suggests the possibility of isolating thin layers starting from bulk $\beta$-antimony crystal following top-down approaches. As commonly done with other 2D materials, we employed the well-known mechanical exfoliation method to prepare thin antimonene flakes starting from a freshly cleaved crystal of $\beta$-antimony (Smart Elements, 99.9999\% purity). It is worth noting that the comparatively short distance between the layers in bulk antimony and the higher strength of the interlayer interaction, makes thin antimony layers extremely difficult to strip-off using the conventional scotch-tape method that is known to work well for the exfoliation of most of 2D materials. We have instead used a commercial high-tack adhesive film to prepare ANSs. First, Sb microcrystals were delaminated by mildly pressing the freshly cleaved surface of the parent $\mathrm{Sb}$ crystals with the adhesive film. The latter is then pressed against a similar piece of adhesive film and after a session of repetitive press-release cycles, ANSs are successfully exfoliated. The exfoliated material is eventually dry-transferred to $\mathrm{a} \mathrm{SiO}_{2} / \mathrm{Si}$ substrate by pressing the adhesive film containing the thin antimony crystallites against the substrate and then gently peeled off. This procedure is further described in detail in the experimental section and schematically illustrated in figure S1 (available online at stacks.iop.org/2DM/8/015018/mmedia). With this strategy we were able to successfully exfoliate ANSs with large areas as displayed in the optical microscopy image in figure $\underline{1}(\mathrm{a})$. It is worth noting that our exfoliation technique yielded a very high density of ANSs having well-defined polygonal shapes with smooth surfaces and sharp edges (figure S1). Such crystalline nanosheets would constitute an excellent scenario to explore fundamental aspects of antimonene, as for example its predicted topological feature [18]. Figure $\underline{1}$ (b) shows a topographic image acquired over the same area shown in figure $\underline{1}(\mathrm{a})$ using AFM revealing thicknesses of several tens of nanometers with 
different terraces as highlighted by the incremental color coding. Figure S2 shows ANSs having well-defined terraces with measured heights of $\sim 1 \mathrm{~nm}$, which fits with monolayer antimonene. Taking into account that traces of the adhesive film under and on top of the flakes are commonplace when using the mechanical exfoliation approach, as well as contributions from effects such as capillary and adhesion forces [35, $\underline{36}$, the apparent heights measured by AFM might be overestimated. Nevertheless, figure $\underline{1}$ (c) shows an AFM image of a representative, isolated thinnest flake, whose height profile indicates an apparent thickness of approximately $14 \mathrm{~nm}$, thus, confirming the successful exfoliation of 2D-antimonene crystals in the few-layer regime. Further AFM topographic images are displayed in figure S3. It is interesting to notice that all the obtained ANSs exhibit different types of polygonal shapes, including, trapezoids, rhombus and triangles. The welldefined shapes of those polygons constitute a first hint of the superior crystallinity within the exfoliated ANSs.

a)

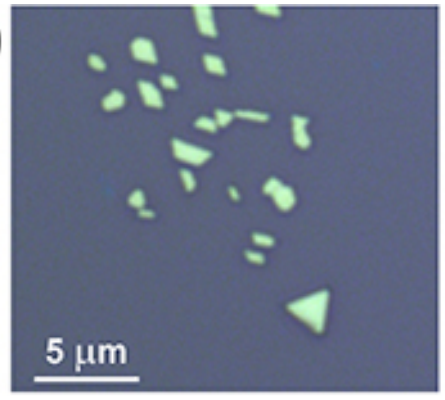

d)

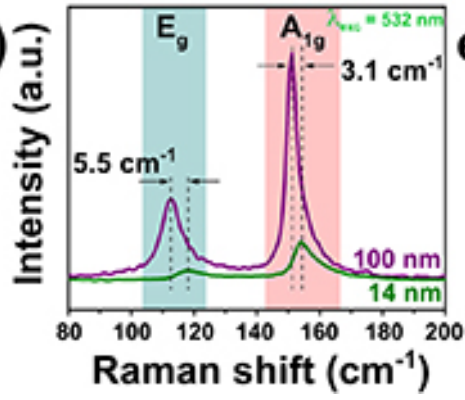

b)

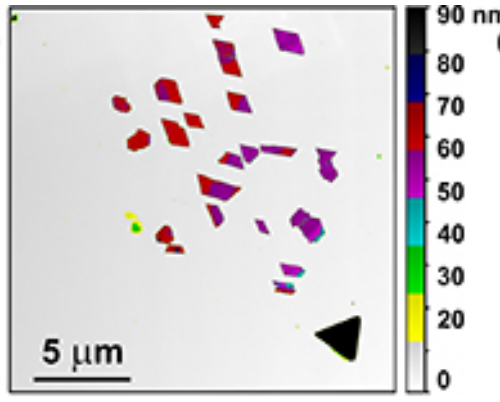

e)

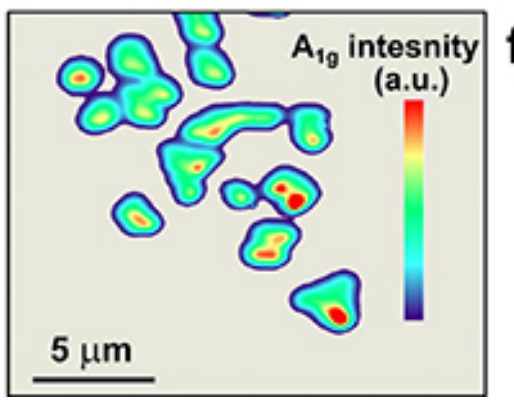

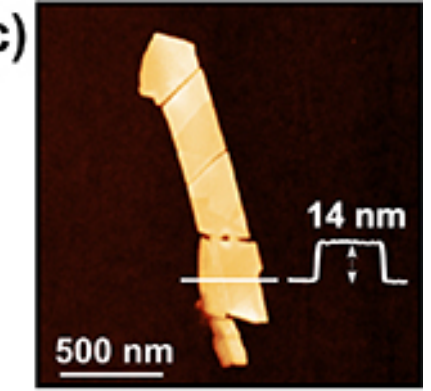
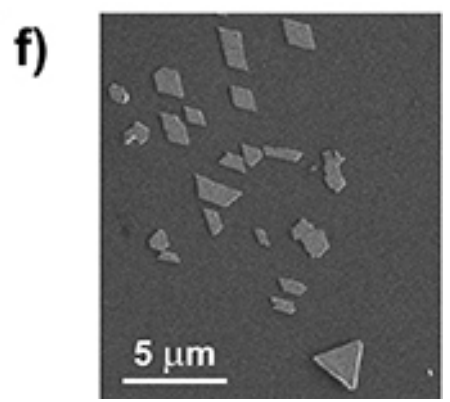

Figure 1. Optical micrograph of typical ANSs isolated on $\mathrm{SiO}_{2} / \mathrm{Si}$ substrate using micromechanical exfoliation. (b) False-colored AFM image of the same ANSs with incremental color code to highlight the terraces. Each color indicates a $10 \mathrm{~nm}$ step (c) AFM image of a fewlayer ANS and height profile along the dashed yellow line. (d) Comparative Raman spectra of the pristine bulk $(100 \mathrm{~nm})$ and few-layer $(14 \mathrm{~nm})$ ANSs depicting a pronounced blue-shift in the peak positions of $A_{1 g}$ and $E_{g}$ modes for the few-layer ANS. (e) Scanning Raman microscopy (SRM) map of the intensity of $A_{1 g}$ mode of the ANSs in (a). ( $f$ ) Scanning electron microscope (SEM) image of the same ANSs. 
In order to investigate the structure of the exfoliated ANSs, we used Raman spectroscopy to probe the crystallinity in a straightforward manner. Figure $\underline{1}(\mathrm{~d})$ shows typical Raman spectra of two ANSs with different thicknesses (100 and $14 \mathrm{~nm}$ ) obtained using an excitation laser with a wavelength of $532 \mathrm{~nm}$. The spectra of the bulk flake (100 $\mathrm{nm}$ ) shows two prominent peaks at $112.6 \mathrm{~cm}^{-1}$ and $151.1 \mathrm{~cm}^{-1}$, corresponding to the vibrations of the crystalline lattice of metallic antimony and are ascribed to the $E_{g}$ and $A_{1 g}$ phonon modes, respectively. The $E_{g}$ mode is attributed to the vibrational mode where the atoms are oscillating in-plane, whereas the $A_{1 g}$ phonon is caused by the out-of-plane vibrations of the sublayers. In case of few-layer ANS (14 nm), the Raman spectra displays a pronounced blue-shift of the $E_{g}$ and $A_{1 g}$ phonon modes compared to the bulk counterpart, which suggests a strong influence of the thickness on the lattice parameter of ANSs. Indeed, recent theoretical calculations have predicted a noticeable phonon shift because of the lattice constant contraction in antimony when the flake thickness is decreased from bulk to mono and few-layer regimes [22]. In our case, the $E_{g}$ mode is blue-shifted by $5.5 \mathrm{~cm}^{-1}$, while the $A_{1 g}$ mode is shifted to a lesser extent by $3.1 \mathrm{~cm}^{-1}$ due to the lower sensitivity of the out-of-plane mode (i.e. $A_{1 g}$ ) to the in-plane lattice constant, in perfect agreement with the theoretical predictions and experimental findings [13]. This effect is believed to be related to the strong electron-lattice interaction and the change in the interlayer interactions as a result of diminished stacking in antimony [37]. We have also conducted a spatially resolved mapping of the $A_{1 g}$ mode intensity using scanning Raman microscopy (SRM) over the same area previously observed by optical microscopy and scanned by AFM (figures $\underline{\underline{1}}(\mathrm{a})-(\mathrm{b})$ ), and the resulting map is shown in figure $\underline{1}(e)$. Raman maps of the $E_{g}$ mode and $A_{1 g} / E_{g}$ ratio are also shown in figure $S 4$. In correlation with the AFM image in figure $\underline{1}$ (b), the Raman map clearly confirms the overall crystal quality within the exfoliated ANSs. SEM was performed to further characterize the morphology of exfoliated ANSs. The SEM micrograph in figure $\underline{1}(\mathrm{f})$ corroborates the results of AFM and Raman measurements and reveals 2D nanosheets having a consistent polygonal morphology with smooth outer surfaces and distinguishable sharp edges, confirming one more time, the high quality of the prepared ANSs. Interestingly, we found that all the isolated ANSs depict exclusively facets of $60^{\circ}$ and $120^{\circ}$ angles, perfectly consistent with the unit cell angle expected from a rhombohedral-lattice system, i.e. $\beta$-antimony (figure S5). Moreover, this particular faceting does indicate that antimony not only undergoes basal cleavage along the c-axis during the mechanical exfoliation process, but also suffers rhombohedral cleavage along preferential/weak crystallographic directions. In fact, it is well known that the greater the difference in bond strength within planes and between planes of a layered material, the easier the cleavage [38]. However, compared to similar 2D materials such as graphene and black 
phosphorus, antimony does not form prototypical van der Waals bonded layered material because of the shorter interlayer distance and the interaction of atomic orbitals between individual double layers which leads to a stronger interlayer adhesion force [39]. Therefore, a relatively difficult exfoliation using top-down approaches is expected. Moreover, the brittleness of antimony adds up an extra level of difficulty which makes exfoliating ultrathin, and at the same time large, antimonene flakes extremely challenging. It partly explains why, to the best of our knowledge, only one example of antimonene nanolayers prepared by mechanical exfoliation can be found in the literature [21]. This observation can be simply exemplified by the multiple fractures observed in the $14 \mathrm{~nm}$ antimonene flake depicted in figure $\underline{1}(\mathrm{c})$. This suggests the existence of a critical thickness enabling the cleavage along weak crystallographic directions, and in the case of thinner antimonene flakes, in competition with the exfoliation along the caxis, leading to flakes with smaller lateral dimensions. We believe that this hypothesis warrants further investigation, which falls beyond the scope of this work.

The chemical composition of our exfoliated ANSs was examined using energy dispersive x-ray (EDX) analysis. Figure S6(a) shows SEM image of an isolated antimony thin flake and the corresponding Sb, O, C and Si EDX mappings. Figure S6(b) shows a uniform distribution of antimony across the whole flake area, while from the color contrast it can be seen that there are negligible traces of oxygen in the flake. Interestingly, we have detected a significant amount of carbon on top of the flake, proving that a residual layer of the polymer from the adhesive film used for the exfoliation is present on the surface [35].

To examine the environmental stability of the as-exfoliated ANSs, we carried out AFM and SRM scans over the exact same flakes shown in figures $\underline{1}(\mathrm{a})$, (b) and (e) after 12 months aging under ambient conditions. Figure S7 confirms the very high stability of antimonene with no structural or topological changes compared to the freshly exfoliated ANSs as confirmed by the Raman mean spectra and AFM height profiles, respectively. It is worth mentioning that, without any prior protection, oxygen species are always detected on the surface of ANSs, although this surface oxidation affects only the top layers yielding a passivating oxide layer that prevents further oxidation [23, 40]. However, when encapsulated by ionic liquids, ANSs showed no sign of oxidation unless this encapsulation is removed and the surface is exposed to air, according to the reported XPS analysis [요 24]. In our case, the above-mentioned residual organic layer on top of the exfoliated ANSs may act as a shield minimizing the extent of surface oxidation to the point where it becomes beyond the detectability limit of our Raman and EDX analysis. 
Temperature-dependent Raman spectroscopy is a powerful measurement method for probing the thermal stability of 2D layered materials as well as understanding their phonon behavior [32]. We conducted an in situ temperature-dependent Raman study in a Linkam heating chamber (THMS 600) where the temperature was controlled between $298 \mathrm{~K}$ and $633 \mathrm{~K}$. Figure $\underline{2}$ (a) illustrates the evolution of mean Raman spectra extracted from spatial mappings of the selected crystal at each temperature set. The laser power on the sample was carefully adjusted and fixed at $0.88 \mathrm{~mW}$ to rule out any possibility of laser-induced damage (figure S8).
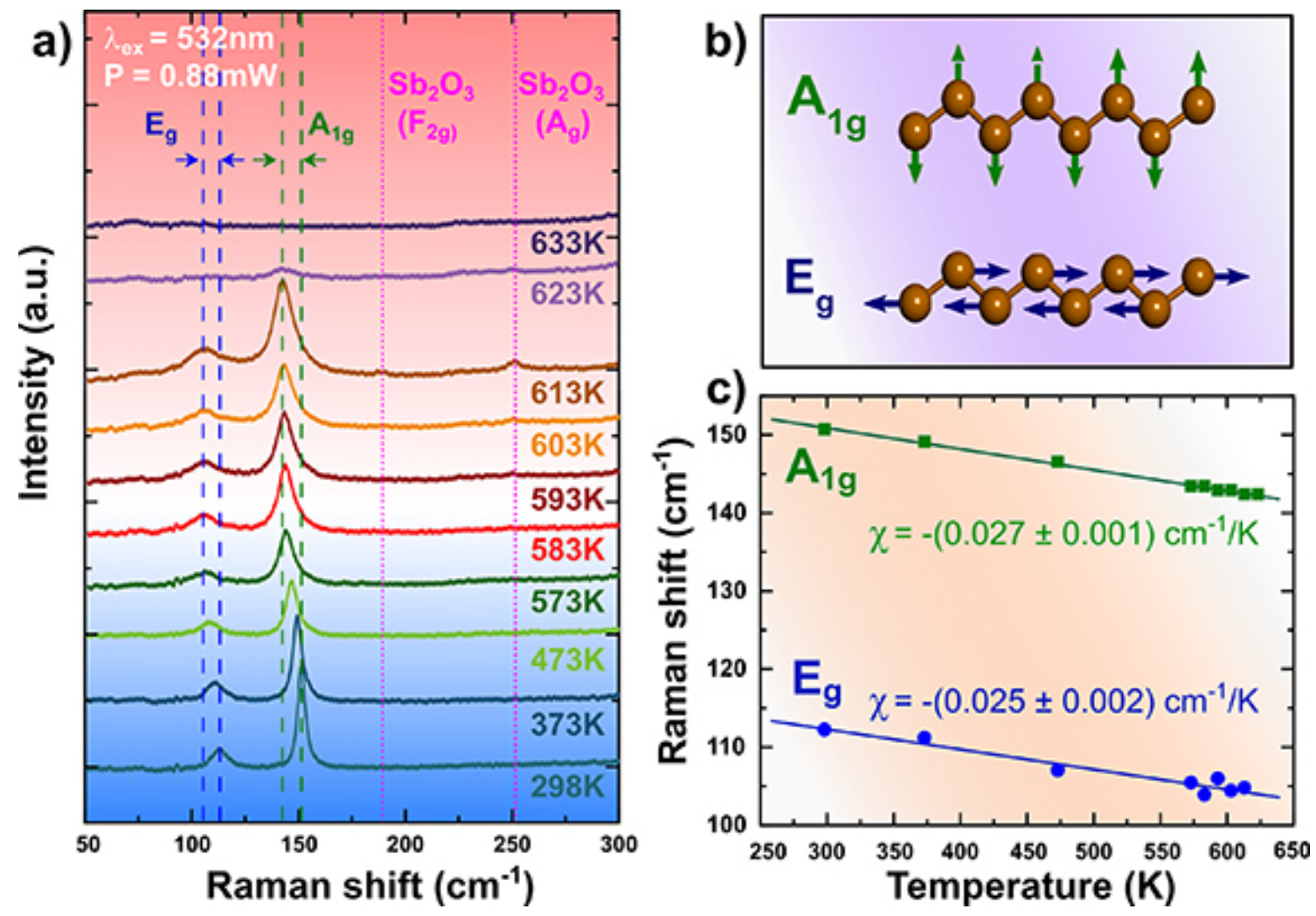

Figure 2. (a) Raman spectra of ANS measured at selected temperatures from 298 to $633 \mathrm{~K}$. Spectra offset vertically for clarity. (b) Schematic diagrams of the two Raman active modes: $A_{1 g}$ and $E_{g}$. The green and blue arrows indicate the direction of the atomic vibrations. (c) Temperature dependence of the Raman peak positions for the $A_{1 g}$ (green squares) and $E_{g}$ (blue circles) modes. Temperature coefficients extracted from the slope of the fit lines (solid lines) to equation (1) for each Raman mode are also shown.

Two noticeable changes can be observed in the Raman spectra evolution. (i) First, we observed the appearance of two new peaks at $250.8 \mathrm{~cm}^{-1}$ and $188.9 \mathrm{~cm}^{-1}$ ascribed, respectively, to ${ }^{1} \mathrm{~A}_{\mathrm{g}}$ and ${ }^{2} \mathrm{~F}_{2 g}$ vibrational modes of the $\alpha$-phase of antimony oxide ( $\alpha$ $\mathrm{Sb}_{2} \mathrm{O}_{3}$ ), when the temperature is increased to $593 \mathrm{~K}$. This observation falls in line with 
the reported low lattice thermal conductivity of antimonene and suggesting a high figure of merit for application in thermoelectric devices [41, 42]. Further increase of temperature to $623 \mathrm{~K}$ resulted in a drastic reduction in the intensity of both antimony and antimony oxide peaks followed by the complete disappearance of every Raman signature at 633 $\mathrm{K}$. This implies that the antimony flake initially undergoes gradual oxidation before eventual sublimation as a result of the heat treatment. The observed oxidation temperature of $593 \mathrm{~K}$ is comparatively lower to the temperature previously reported for the oxidation of CVD-grown antimony flakes [26]. The underlying reason of such difference might be the presence of residual viscoelastic polymer under and/or on top of the flake, as we mentioned earlier, and the heating procedure by which our temperaturedependent Raman measurement has been carried out (i.e. lower heating rate of 10 $\mathrm{K} . \mathrm{min}^{-1}$, followed by 5 min waiting to allow for thermal stabilization of the sample and optics). (ii) Second, an evident downshifting (red-shift) and broadening of both $\mathrm{A}_{1 \mathrm{~g}}$ and $E_{g}$ peaks can be easily noticed as the temperature increases. Using a Lorentzian peak fitting, we extracted the positions of the $A_{1 g}$ and $E_{g}$ peaks and plotted them against temperature in figure 2(c). A clear linear dependence of the phonon shift on the temperature is observed for both $A_{1 g}$ and $E_{g}$ modes. Generally, the linear variation in Raman modes position with temperature can be assigned to the anharmonic vibrations of the lattice, which involves contributions from the lattice thermal expansion to the interatomic potential energy, mediated by phonon-phonon interactions [43]. As the lattice expands or contracts because of temperature change, the equilibrium positions of atoms and consequently the interatomic forces change accordingly, which induces shifts in the phonon energies $[\underline{32}, \underline{44}]$.

To gain further understanding of the observed linear decrease in phonon energy, we adopted a well-established approach by fitting our experimental data with equation (1 $)$ [45],

$\omega(T)=\omega_{0}+\chi T$

Where $\omega_{0}$ is the phonon frequency at a temperature interpolated to $0 \mathrm{~K}$ and $\chi$ is the firstorder temperature coefficient of the corresponding Raman mode defining the slope of the dependence $[44, \underline{46}]$. Our experimental values for each mode together with values for other $2 \mathrm{D}$ materials from literature are listed in table $\underline{1}$. The $\chi$ values for $A_{1 g}$ and $E_{g}$ modes are 0.027 and $0.025 \mathrm{~cm}^{-1} \mathrm{~K}^{-1}$, respectively, implying the same temperature sensitivity of both modes as well as a similar electron-phonon coupling in ANSs [47]. These values are comparable to that of ultrathin $\mathrm{Sb}_{2} \mathrm{O}_{3}$ [48], higher than graphene [32], $\mathrm{MoS}_{2}$ [49] and BP [33], while it is smaller than that of SnSe [50]. Previous works have revealed that the first-order temperature coefficient of the Raman modes in layered 
materials is associated to the van der Waals interaction between each layer [ $\underline{51}, \underline{52}]$. For instance, in graphene and $\mathrm{MoS}_{2}$ the weak van der Waals interlayer interaction leads to the small $X$ values, whereas in BP and SnSe, the van der Waals interaction is strong, leading to large $X$ value. In our case, the individual layers of antimony are held together by an adhesion force stronger than weak van der Waals interaction, hence the measured large $X$ values.

Table 1. First-order temperature coefficients $x$ obtained from temperature-dependent Raman spectra in this work and for other 2D materials.

\begin{tabular}{|c|c|c|c|c|}
\hline materials & $\lambda_{e x c}(\mathrm{~nm})$ & $\begin{array}{l}\text { Raman } \\
\text { modes }\end{array}$ & $X\left(\mathrm{~cm}^{-1} \mathrm{~K}^{-1}\right)$ & References \\
\hline Sb nanosheets & 532 & $A_{1 g} E_{g}$ & $-0.027-0.025$ & This work \\
\hline $\mathrm{Sb}_{2} \mathrm{O}_{3}$ & 532 & $\alpha-{ }^{1} A_{g} \beta-A_{g}$ & $-0.025-0.023$ & {$[48]$} \\
\hline $\begin{array}{l}\text { Monolayer } \\
\text { graphene Bilayer } \\
\text { graphene }\end{array}$ & 488 & G mode & $-0.016-0.015$ & [47] \\
\hline $\mathrm{MoS}_{2}$ & 532 & $A_{1 g} E_{2 g}{ }^{1}$ & $-0.012-0.013$ & [49] \\
\hline $\mathrm{BP}$ & 532 & $A_{1 g} A_{2 g} B_{2 g}$ & $\begin{array}{l}-0.010-0.014- \\
0.013\end{array}$ & [33] \\
\hline SnSe & 532 & $B_{3 g} A_{g 2} A_{g 3}$ & $\begin{array}{l}-0.033-0.037- \\
0.015\end{array}$ & [50] \\
\hline
\end{tabular}

It is known that lasers focused on a small area can significantly increase the local temperature of the sample, which enables the study of photothermal effects in 2D materials [53]. In order to achieve a comprehensive understanding of the intrinsic photothermal property of our exfoliated ANSs, we carried out a laser power-dependent Raman study of which the experimental setup is schematically represented in figure $\underline{3}(a)$. The selected ANS was illuminated by a focused laser beam in gradually increasing 
power spanning from 0.14 to $14 \mathrm{~mW}$. The corresponding Raman spectra shown in figure $\underline{3}$ (b) were collected using a $532 \mathrm{~nm}$ excitation laser and a $100 \times$ objective (NA = $0.8)$ in the Horiba LabRAM HR Evolution microscope. As the laser power increases, both $E_{g}$ and $A_{1 g}$ modes shift to lower wavenumber values indicating a similar anharmonic vibrations of the lattice as previously observed in the temperature-dependent Raman study, in this case due to laser heating. Remarkably, when the laser power is raised to $14 \mathrm{~mW}$, typical Raman modes of $\mathrm{\alpha}-\mathrm{Sb}_{2} \mathrm{O}_{3}\left({ }^{1} \mathrm{~A}_{\mathrm{g}}\right.$ and $\left.{ }^{2} \mathrm{~F}_{2 \mathrm{~g}}\right)$ start to emerge, indicating a laser-induced oxidation of the ANS as a result of the local heating. A further gradual decrease of the laser power demonstrates that the induced oxidation is permanent (figure S9). This finding reveals the outstanding photothermal conversion efficiency of antimonene, one of the highest among the 2D materials family according to recent report [54], thus highlighting its high potential for applications in photothermal cancer therapy [55]. Furthermore, the observed partial oxidation is of critical importance as it can promote the photothermal conversion efficiency even further and improve its efficacy in biomedical applications thanks to the high toxicity of the generated antimony oxide layer [56, 57]. Interestingly, the measured red-shifts in $A_{1 g}$ and $E_{g}$ modes follow a linear dependency with the laser power (figure $\underline{3}(\mathrm{~d})$ ). In practice, by taking advantage of the sensitive response of phonon frequencies in atomically thick materials to local heating by laser excitation, it is possible to perform optothermal thermometry measurements based on Raman spectroscopy. For example, local temperature and thermal conductivity of several atomically thick materials have been successfully determined, in situ, both on supported and suspended 2D crystals [ $[\underline{58}, \underline{59}$. In our case, using the thermal and power coefficients extracted from the temperature and laser power-dependent Raman measurements, we have been able to estimate the temperature of the probed ANS (figure $\underline{3}(e)$ ). However, careful attention should be paid as the experimental measurements tend to be overestimated when this approach is performed on a surface [60]. Therefore, in order to better understand the heat transfer mechanism in our exfoliated ANSs, we have developed a reliable model using COMSOL Multiphysics that additionally offers the possibility to illustrate the different reasons of such a systematic overestimation. 
a)

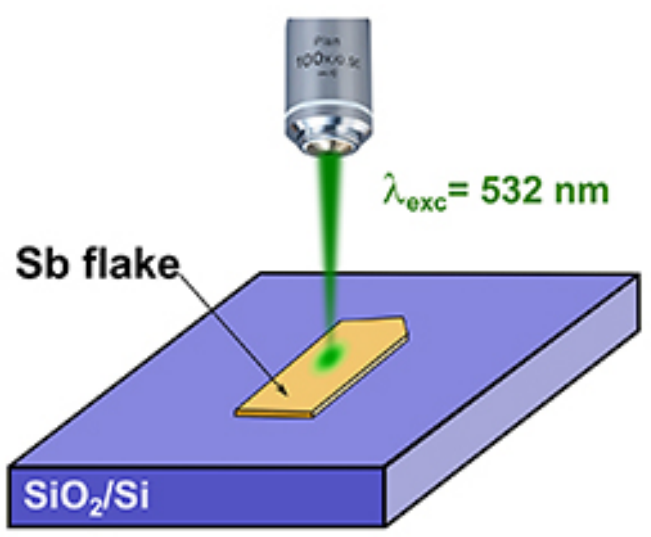

b)

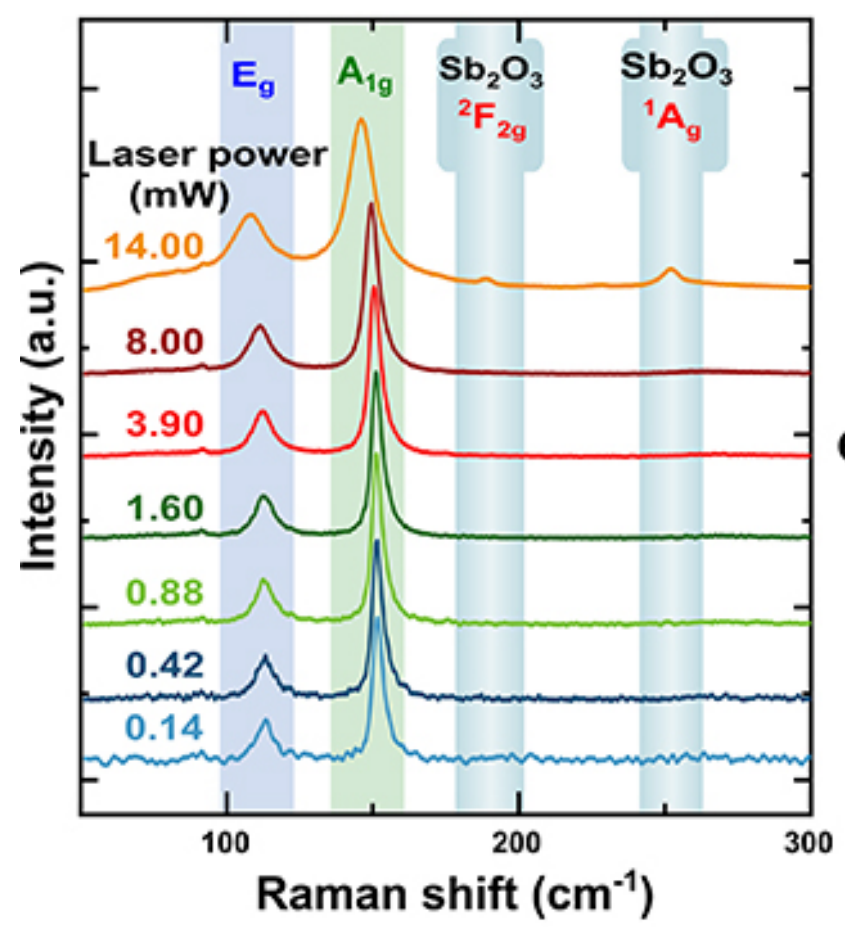

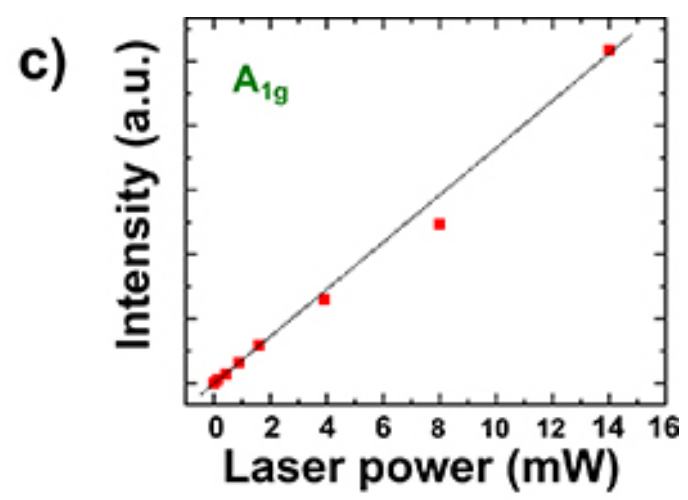

d)

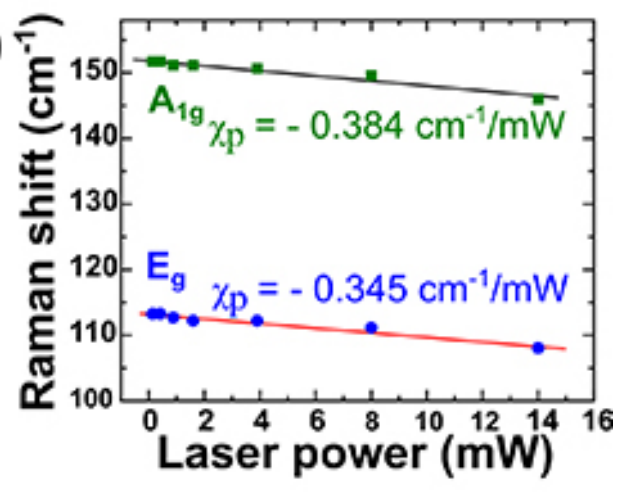

e)

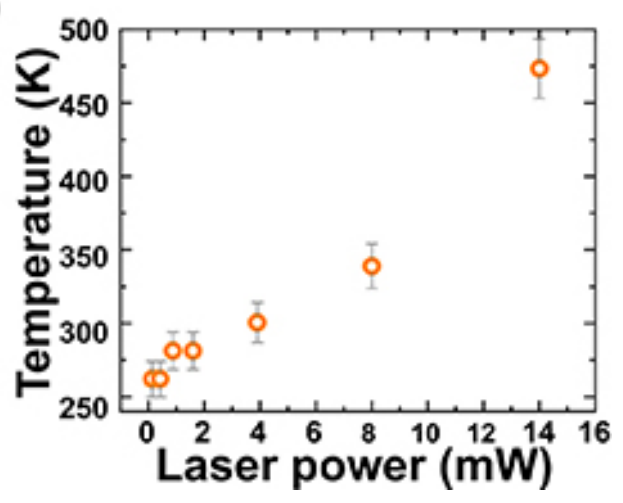

Figure 3. (a) Schematic of the laser power-dependent Raman measurement showing supported ANS on $\mathrm{SiO}_{2} / \mathrm{Si}$ and the laser excitation wavelength. A Raman laser beam is simultaneously causing and probing the local temperature increase on the sample (b) Normalized Raman spectra of ANS measured at increasing excitation laser power from 0.14 to $14 \mathrm{~mW}$ ( $\lambda_{\mathrm{exc}}=532 \mathrm{~nm}$ ) at RT in air. Spectra offset vertically for clarity. (c) Linear increase of the intensity of the $A_{1 g}$ mode as a function of the incident laser power. (d) Power coefficients, $\chi_{p}$, for both two modes (green squares and blue circles for $A_{1 g}$ and $E_{g}$, respectively) are extracted from the slope of the linear fits (solid lines). (e) Temperature of the probed ANS as a function of laser power, estimated using both calculated power and temperature coefficients.

We consider a disk-like heat source embedded into a $40 \mathrm{~nm}$ thick ANS laying on the substrate, i.e. a low aspect ratio disk of $285 \mathrm{~nm}$ height in contact to the silicon wafer. The silicon is introduced as a semi-spherical bulk to avoid border effects. We calculate the 
stationary temperature distribution solving the Fourier equation for this geometry in conditions of thermal isolation. In these conditions, we observe three important findings: i) the silicon behaves as a heat sink, ii) the silica limits the thermal energy transfer towards the substrate, and as a result iii), the heat is concentrated around the excitation spot. A study on the influence of the different experimental parameters on the temperature contrast is provided as Supporting Information (figures S10-13). Our model allows to connect our experiment with previous results in literature, which are essential to understand the high temperature increase in our samples under laser excitation as well as for discussing the oxidation mechanisms of the upper layer $[\underline{53}, \underline{60}]$.

In figure $\underline{4}(\mathrm{a})$ we present a zenith view of a mapping corresponding to a rectangular flake $\left(2 \times 4 \mu \mathrm{m}^{2}\right)$ embedding a $1 \mu \mathrm{m}$ diameter heat source of $1 \mathrm{~mW}$. The borders of the flake are kept at or close to RT, as the heat is strongly concentrated around the source, as predicted by our model. This is also shown in the transversal cross sections [figures $\underline{4}$ (b) and (c)] where we can observe the temperature increase in $\mathrm{SiO}_{2}$ and the efficiency of silicon as a heat sink. The low thermal conductivity of silica together with the silicon heat sink leads to a linear dependence of the temperature on the excitation power, shown in figure $\underline{4}(\mathrm{~d})$. The slope of this curve would be inversely proportional to the thermal conductivity of the flake. The scatters in this plot correspond to the experimental measurement in figure $\underline{3}(e)$ for a flake with dimensions similar to those of our simulations. As in previous reports, the values of the thermal conductivity on a surface are overestimated with respect to theoretical calculations as most of the experimental points lie on the curve of $K_{S b}=90 \mathrm{~W} \cdot \mathrm{m}^{-1} \mathrm{~K}^{-1}[\underline{41}, \underline{61]}$. 


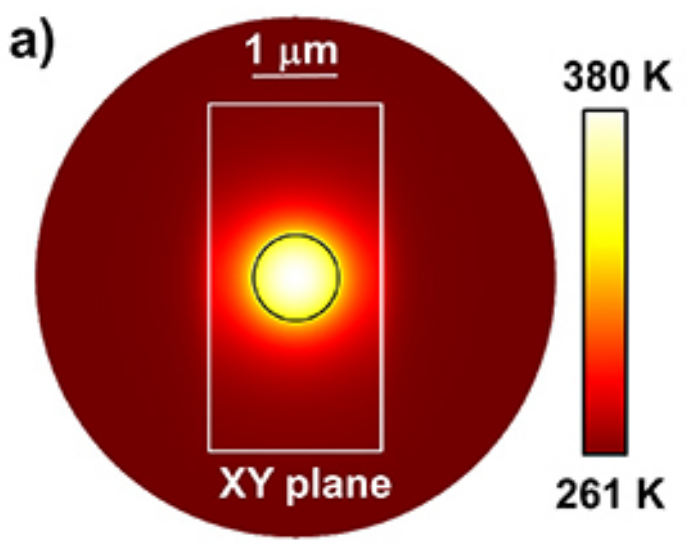

b)
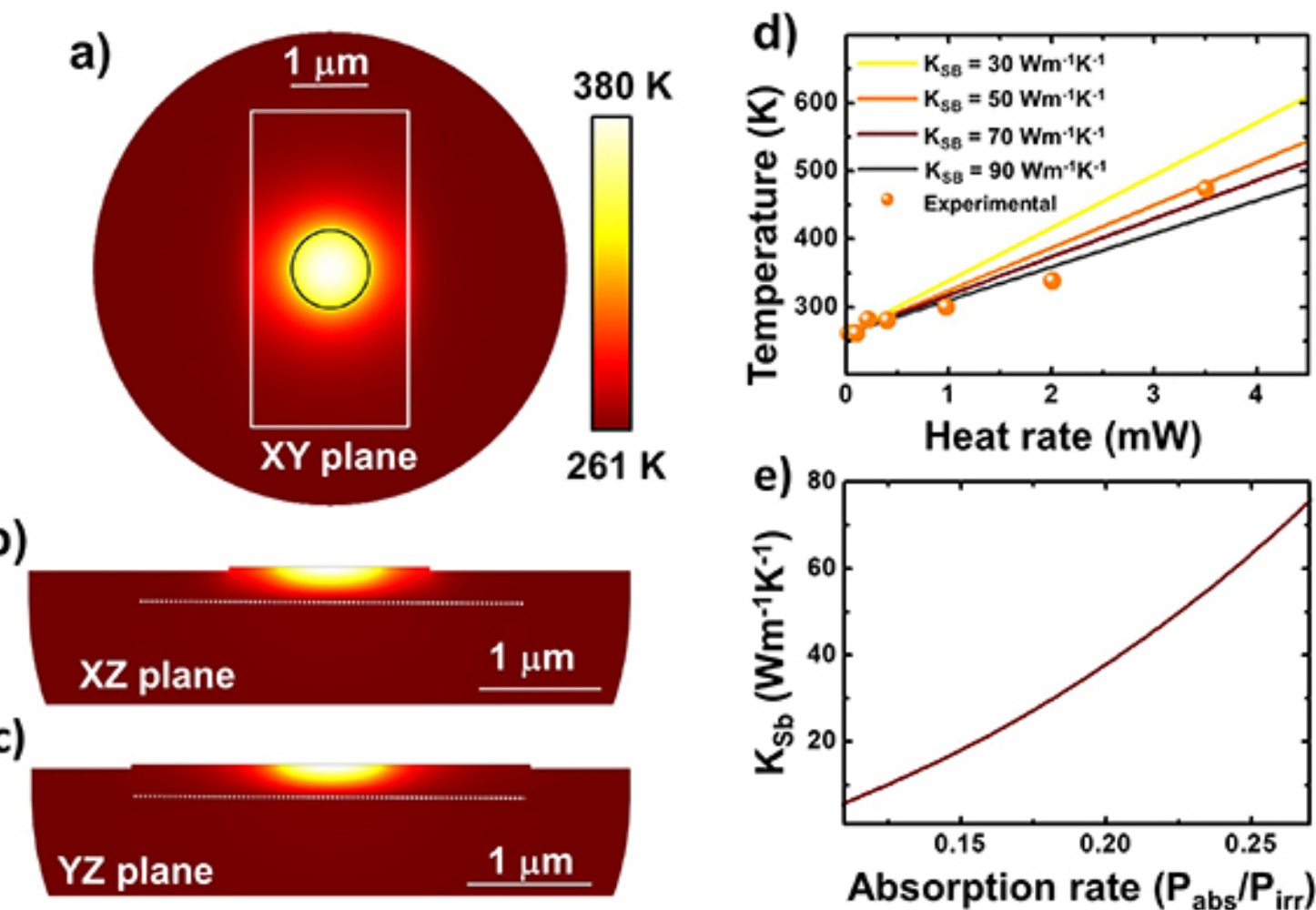

Absorption rate $\left(\mathbf{P}_{\text {abs }} / \mathbf{P}_{\text {irr }}\right)$

Figure 4. (a) Zenith view of the temperature mapping for a rectangular flake (white lines) considering a disk-like heat source in to the black circle. Figures (b) and (c) represent cross sections of the temperature mapping. The silica-silicon interface is delimited by a dot line. (d) Average temperature into the excitation spot as a function of the heat source rate for different thermal conductivities of the flake. The experimental data (open dots) are included by considering an absorption efficiency of $25 \%$ of the excitation laser light at the flake. (e) Impact of the experimental determination of the thermal conductivity $\mathrm{K}_{\mathrm{sb}}$ as a function of the absorption rate.

Based on our model we can weigh the possible reasons of the systematic overestimation of the thermal conductivity values in flakes deposited on a surface. Firstly, we are neglecting convection loss which in the field of 2D materials are always assumed to be low [58], however, it is worth mentioning that convection can introduce a factor-two deviation in methods employed for bulk materials. Secondly, a more accurate description of the laser spot as thermal source is required. For example, we have estimated an excitation spot of $1 \mu \mathrm{m}$ with an effective absorbance of around $30 \%$ for a $40 \mathrm{~nm}$ thickflake using the refractive index data provided by Ares et al [62]. Slight deviations with respect to these values would affect dramatically the slope of our experimental data in figure $\underline{4}(\mathrm{~d})$. This is shown in figure $\underline{4}(\mathrm{e})$ where we plot the value $K_{S b}$ extracted from the experimental data in figure $\underline{4}(\mathrm{~d})$ for different absorption rates. Thirdly, experimental parameters such as the thermal conductivity of silica and silicon should not be as important as optical considerations, see Supporting Information. Finally, we want to point 
out that the values obtained by means of photothermal methods will correspond to high temperature values, necessarily higher than room temperature values. This detail might seem naïve, but it can cause serious confusion for a non-specialized reader as it is not emphasized in most papers on this topic.

As aforementioned, the accurate estimation of the thermal conductivity and heat capacity would require a better description of the laser excitation in terms of a heat source, thus falling beyond the scope of the present work [63]. Instead, we conducted similar highpower irradiation experiments using different excitation wavelengths (457, 473, 633 and $785 \mathrm{~nm}$ ). Based on the intensity of $\mathrm{Sb}_{2} \mathrm{O}_{3}{ }^{\prime}{ }^{1} \mathrm{~A}_{\mathrm{g}}$ peak (figure S14), we found that surface oxidation is more efficient using shorter wavelength lasers (457 and $473 \mathrm{~nm}$ ), while longer wavelengths lasers induced mild (633 nm) or no oxidation $(785 \mathrm{~nm})$. In connection to our heat transfer model, this could be explained either by a variation of the absorption efficiency or by an increase of the excitation spot with the excitation wavelength. However, an estimation of the absorption efficiency using the Transfer Matrix Method indicates that there is a local minimum in absorption around 450-500 nm (see figure S15), which underscores the importance of the proper determination of the material's refractive index for a quantitative discussion.

The laser-induced oxidation so far observed warrants additional characterization to understand the changes produced in the studied ANSs from the structural and topological points of view. For that purpose, we analyzed the structure and topology of an ANS before and after high power laser irradiation (14 mW, for $10 \mathrm{~s}$ ) using both SRM and AFM. In these conditions not only does the laser beam induced oxidation but also triggered some serious damage on the irradiated ANS (figures $\underline{5}(\mathrm{a})-(\mathrm{d})$ ). A hole of approximately $40 \mathrm{~nm}$ (figure S16), which is also visible in the SEM image of 4(e), was created accompanied by the onset of the ${ }^{1} \mathrm{~A}_{\mathrm{g}}$ and ${ }^{2} \mathrm{~F}_{2 \mathrm{~g}}$ Raman modes of $\alpha-\mathrm{Sb}_{2} \mathrm{O}_{3}$ (see also figure S16 (e)-(f) and (h)). EDX elemental mapping of a similarly irradiated ANS with high-power laser beam depicts a local decrease in the intensity of antimony and a decrease in the silicon intensity from the underlying substrate (figure S17). 

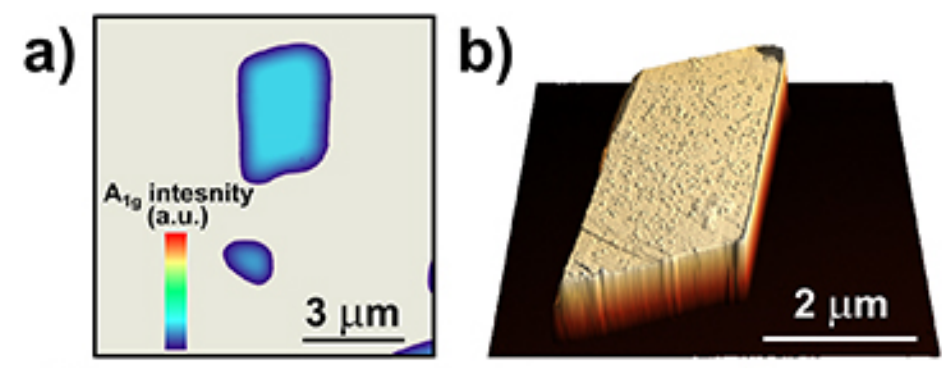

e) laser-damaged area

c)

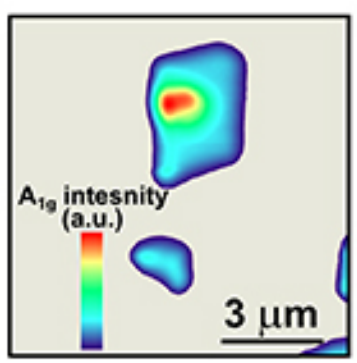

d)

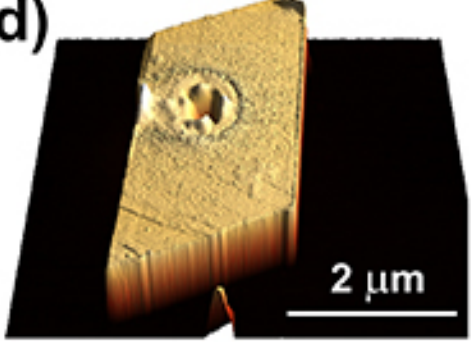

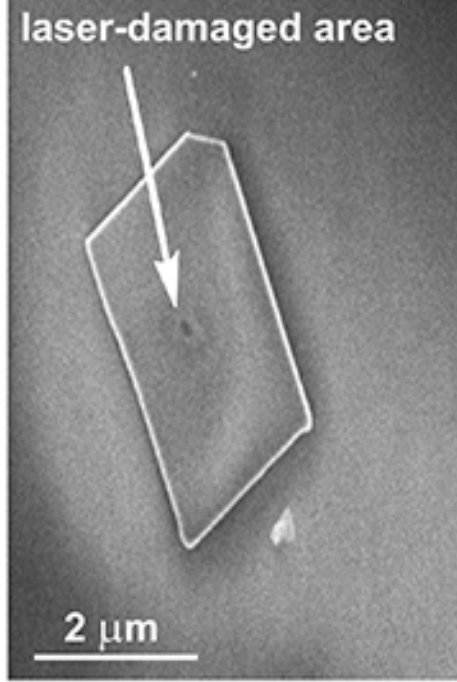

Figure 5. (a) SRM map of $A_{1 g}$ peak intensity and (b) 3D topographic AFM picture of isolated ANS $\left(40 \mathrm{~nm}\right.$ ) before and after (c) and (d) high-power laser irradiation ( $\lambda_{\text {exc }}=532 \mathrm{~nm}$ at $14 \mathrm{~mW}$ for 10 s), respectively. (e) SEM image of the same irradiated ANS showing the laser-damaged area.

Given the thermoelectric capabilities of the $\mathrm{Sb}$ and $\mathrm{\alpha}-\mathrm{Sb}_{2} \mathrm{O}_{3}$, it is of interest to exploit this process in order to improve the control on the photo-oxidation of antimonene for developing local oxidation photolithography applications. As an example of the potential of this approach we have carried out preliminary experiments where we performed a spatially resolved Raman mapping of an isolated ANS using a lower dose of laser power (3.9 $\mathrm{mW}$ for $10 \mathrm{~s}$ ), just enough to intentionally induce controlled oxidation on the surface of the flake without damaging its structural integrity (figure S19).

By analyzing the height profiles acquired along the studied ANS, before and after laser irradiation, we observe small spots of laser-deposited material that show certain increase in height at the edges compared to the center area (see figure S120 (c) and (d)). This provides strong evidence that the oxidation of antimonene starts preferentially from the edges, and revealing that a spatially controlled laser-induced oxidation of antimonene on the sub- $\mu \mathrm{m}$ scale is challenging but reliable. In principal, arbitrary heterostructures of $\mathrm{Sb}_{2} \mathrm{O}_{3}$ /antimonene could be realized, as theoretically predicted and experimentally suggested in our recent reports $[40,64]$. Such exotic structures based on antimonene are in fact of great importance both from the fundamental and technological points of view, as they can induce radical changes in the physical and chemical properties of the original flake. For instance, surface oxidation of antimonene nanostructures was reported to open an optical bandgap [40], promote the cytotoxicity to cancer cells [56] and even improve the catalytic activity towards different reactions $[23, \underline{65}$. Therefore, developing a reliable method to both produce and control surface oxidized antimonene- 
based structures is highly sought-after. Our results confirm that it can be experimentally realized, although a more sophisticated photolithography technique and thinner antimonene flakes would be required.

\section{Conclusion}

In conclusion, we introduced a deterministic method to isolate ANSs through micromechanical exfoliation. We reported the first experimental investigation of the temperature dependence of the frequencies of both $A_{1 g}$ and $E_{g}$ modes in the Raman spectra of ANSs on $\mathrm{SiO}_{2} / \mathrm{Si}$ substrates. A linear temperature-dependence of the position of the Raman modes was observed and explained by the phenomenon of anharmonic lattice vibrations mediated by phonon-phonon interactions. First-order temperature coefficients were extracted and are $X=-0.027$ and $X=-0.025 \mathrm{~cm}^{-1} \mathrm{~K}^{-1}$ for $\mathrm{A}_{1 \mathrm{~g}}$ and $E_{g}$ modes, respectively. Laser-induced oxidation of the exfoliated ANS was demonstrated and described by a combination of laser power- and wavelengthdependent studies with a numerical heat transfer model. Despite the difficulty of quantifying the thermal properties of our system, relevant information about the oxidation process in antimonene flakes could be obtained. Our results provide fundamental information about the heat transfer at the nanoscale and the oxidation susceptibility of 2D antimonene, which is crucial for understanding its thermophysical properties as well as for developing advanced applications in fields such as photothermal cancer therapy and catalysis.

\section{Acknowledgments}

The authors thank the European Research Council (ERC Starting Grant 804110 2DPnictoChem to G.A.) for financial support. G.A. thanks the financial support from the Generalitat Valenciana (CIDEGENT/2018/001 grant and iDiFEDER/2018/061 cofinanced by FEDER), the Spanish MICINN (PID2019-111742GA-I00 and Excellence Unit Maria de Maeztu (CEX2019-000919-M)), and the Deutsche Forschungsgemeinschaft (DFG, FLAG-ERA AB694/2-1). J.C.-F. thanks the financial support from Generalitat Valenciana (CIDEGENT/2018/005). Authors thank Dr. C. Gibaja and I. Torres for their assistance with the micromechanical exfoliation procedure and $\mathrm{E}$. Weinreich for the technical support with laser-induced oxidation Raman measurements. 


\section{Conflict of interest}

The authors declare no conflict of interest.

\section{References}

1. Novoselov K S, Geim A K, Morozov S V, Jiang D, Zhang Y, Dubonos S V, Grigorieva I V and Firsov A A 2004 Electric field effect in atomically thin carbon films Science 306 666-9

2. Kubota Y, Watanabe $K$, Tsuda $O$ and Taniguchi T 2007 Deep ultraviolet lightemitting hexagonal boron nitride synthesized at atmospheric pressure Science 317 932-4

3. Chhowalla M, Shin H S, Eda G, Li L-J, Loh K P and Zhang H 2013 The chemistry of two-dimensional layered transition metal dichalcogenide nanosheets Nat. Chem. 5 263-75

4. Wang Q H, Kalantar-Zadeh K, Kis A, Coleman J N and Strano M S 2012 Electronics and optoelectronics of two-dimensional transition metal dichalcogenides Nat. Nanotechnol. 7 699-712

5. Kashid R V, Late D J, Chou S S, Huang Y-K, De M, Joag D S, More M A and Dravid V P 2013 Enhanced field-emission behavior of layered MoS2 sheets Small 9 2730-4

6. Zhang S, Xie M, Li F, Yan Z, Li Y, Kan E, Liu W, Chen Z and Zeng H 2016 Semiconducting group 15 monolayers: a broad range of band gaps and high carrier mobilities Angew. Chem., Int. Ed. 55 1666-9

7. Castellanos-Gomez A et al 2014 Isolation and characterization of few-layer black phosphorus 2D Mater. 1025001

8. Lloret $V$ et al 2019 Few layer 2D pnictogens catalyze the alkylation of soft nucleophiles with esters Nat. Commun. 10509

9. Tejeda-Serrano M, Lloret $V, B G M$, Simon $F$, Hauke $F$, Hirsch A, DoménechCarbó A, Abellán G and Leyva-Pérez A 2020 Few-layer black phosphorous catalyzes radical additions to alkenes faster than low-valence metals ChemCatChem 12 2226-32

10. Niu X, Yi Y, Meng L, Shu H, Pu Y and Li X 2019 Two-dimensional phosphorene, arsenene, and antimonene quantum dots: anomalous sizedependent behaviors of optical properties J. Phys. Chem. C 123 25775-80

11. Huang Y, Zhu C, Zhang S, Hu X, Zhang K, Zhou W, Guo S, Xu F and Zeng H 2019 Ultrathin bismuth nanosheets for stable Na-ion batteries: clarification of structure and phase transition by in situ observation Nano Lett. 19 1118-23

12. Zhang S, Zhou W, Ma Y, Ji J, Cai B, Yang S A, Zhu Z, Chen Z and Zeng $H$ 2017 Antimonene oxides: emerging tunable direct bandgap semiconductor and novel topological insulator Nano Lett. 17 3434-40 
13. Ji J et al 2016 Two-dimensional antimonene single crystals grown by van der Waals epitaxy Nat. Commun. 713352

14. Abellán G, Wild S, Lloret V, Scheuschner N, Gillen R, Mundloch U, Maultzsch J, Varela M, Hauke F and Hirsch A 2017 Fundamental insights into the degradation and stabilization of thin layer black phosphorus J. Am. Chem. Soc. 139 10432-40

15. Ares P, Palacios J J, Abellán G, Gómez-Herrero J and Zamora F 2018 Recent progress on antimonene: a new bidimensional material Adv. Mater. 301703771

16. Zhang S, Yan Z, Li Y, Chen Z and Zeng H 2015 Atomically thin arsenene and antimonene: semimetal-semiconductor and indirect-direct band-gap transitions Angew. Chem., Int. Ed. 54 3112-5

17. Kripalani D R, Kistanov A A, Cai Y, Xue M and Zhou K 2018 Strain engineering of antimonene by a first-principles study: mechanical and electronic properties Phys. Rev. B 98085410

18. Zhao M, Zhang $X$ and Li L 2015 Strain-driven band inversion and topological aspects in Antimonene Sci. Rep. 516108

19. Lee J, Tian W-C, Wang W-L and Yao D-X 2015 Two-dimensional pnictogen honeycomb lattice: structure, on-site spin-orbit coupling and spin polarization Sci. Rep. 511512

20. Wang G, Pandey R and Karna S P 2015 Atomically thin group V elemental films: theoretical investigations of antimonene allotropes ACS Appl. Mater. Interfaces 7 11490-6

21. Ares $P$, Aguilar-Galindo $F$, Rodríguez-San-Miguel $D$, Aldave $D$ A, Díaz-Tendero S, Alcamí M, Martín F, Gómez-Herrero J and Zamora F 2016 Mechanical isolation of highly stable antimonene under ambient conditions Adv. Mater. 28 6332-6

22. Gibaja C et al 2016 Few-layer antimonene by liquid-phase exfoliation Angew. Chem., Int. Ed. 55 14345-9

23. Gibaja C et al 2019 Liquid phase exfoliation of antimonene: systematic optimization, characterization and electrocatalytic properties J. Mater. Chem. A 7 22475-86

24. Abellán $G$ et al 2017 Noncovalent Functionalization and Charge Transfer in Antimonene Angew. Chem., Int. Ed. 56 14389-94

25. Sun $X$, Lu Z, Xiang $Y$, Wang $Y$, Shi J, Wang G-C, Washington M A and Lu T-M 2018 van der waals epitaxy of antimony islands, sheets, and thin films on single-crystalline graphene ACS Nano 12 6100-8

26. Wu Q and Y J S 2018 The environmental stability of large-size and singlecrystalline antimony flakes grown by chemical vapor deposition on $\mathrm{SiO} 2$ substrates Chem. Commun. 54 9671-4 
27. Niu T, Zhou W, Zhou D, Hu X, Zhang S, Zhang K, Zhou M, Fuchs H and Zeng $H 2019$ modulating epitaxial atomic structure of antimonene through interface design Adv. Mater. 311902606

28. Peng L, Ye S, Song J and Qu J 2019 Solution-phase synthesis of few-layer hexagonal antimonene nanosheets via anisotropic growth Angew. Chem., Int. Ed. 58 9891-6

29. Balandin A A 2011 Thermal properties of graphene and nanostructured carbon materials Nat. Mater. 10 569-81

30. Cai W, Moore A L, Zhu Y, Li X, Chen S, Shi L and Ruoff R S 2010 Thermal transport in suspended and supported monolayer graphene grown by chemical vapor deposition Nano Lett. 10 1645-51

31. Sahoo S, Chitturi V R, Agarwal R, Jiang J-W and Katiyar R S 2014 Thermal conductivity of freestanding single wall carbon nanotube sheet by raman spectroscopy ACS Appl. Mater. Interfaces 6 19958-65

32. Calizo I, Balandin A A, Bao W, Miao F and Lau C N 2007 Temperature dependence of the raman spectra of graphene and graphene multilayers Nano Lett. 7 2645-9

33. Late D J 2015 Temperature dependent phonon shifts in few-layer black phosphorus ACS Appl. Mater. Interfaces 7 5857-62

34. Xia J, Li X-Z, Huang X, Mao N, Zhu -D-D, Wang L, Xu H and Meng X-M 2016 Physical vapor deposition synthesis of two-dimensional orthorhombic SnS flakes with strong angle/temperature-dependent Raman responses Nanoscale 8 2063-70

35. Schweizer P, Dolle C, Dasler D, Abellán G, Hauke F, Hirsch A and Spiecker E 2020 Mechanical cleaning of graphene using in situ electron microscopy Nat. Commun. 111743

36. Nemes-Incze P, Osváth Z, Kamarás K and Biró L P 2008 Anomalies in thickness measurements of graphene and few layer graphite crystals by tapping mode atomic force microscopy Carbon 46 1435-42

37. Wang X, Kunc K, Loa I, Schwarz U and Syassen K 2006 Effect of pressure on the Raman modes of antimony Phys. Rev. B 74134305

38. Brian M and Berry L G 1968 Elements of Mineralogy (San Francisco: W.H. Freeman) 115-7

39. Pumera M and Sofer Z 2017 2D monoelemental arsenene, antimonene, and bismuthene: beyond black phosphorus Adv. Mater. 291605299

40. Assebban $M$ et al 2020 Unveiling the oxidation behavior of liquid-phase exfoliated antimony nanosheets 2D Mater. 7025039

41. Sharma S, Kumar S and Schwingenschlögl U 2017 Arsenene and antimonene: two-dimensional materials with high thermoelectric figures of merit Phys. Rev. Appl. 8044013 
42. Wang S, Wang $W$ and Zhao G 2016 Thermal transport properties of antimonene: an ab initio study Phys. Chem. Chem. Phys. 18 31217-22

43. Balkanski $M$, Wallis $R F$ and Haro $E 1983$ Anharmonic effects in light scattering due to optical phonons in silicon Phys. Rev. B 28 1928-34

44. Pawbake A S, Pawar M S, Jadkar S R and Late D J 2016 Large area chemical vapor deposition of monolayer transition metal dichalcogenides and their temperature dependent Raman spectroscopy studies Nanoscale 8 3008-18

45. Zouboulis E S and Grimsditch M 1991 Raman scattering in diamond up to 1900 K Phys. Rev. B 43 12490-3

46. Lanzillo N A et al 2013 Temperature-dependent phonon shifts in monolayer MoS2 Appl. Phys. Lett. 103093102

47. Late D J, Maitra U, Panchakarla L S, Waghmare U V and Rao C N R 2011 Temperature effects on the Raman spectra of graphenes: dependence on the number of layers and doping J. Phys.: Condens. Matter 23055303

48. Han W et al 2019 Two-dimensional inorganic molecular crystals Nat. Commun. 104728

49. Sahoo S, Gaur A P S, Ahmadi M, Guinel M J-F and Katiyar R S 2013 Temperature-dependent raman studies and thermal conductivity of few-layer MoS2 J. Phys. Chem. C 117 9042-7

50. Luo S, Qi X, Yao H, Ren X, Chen Q and Zhong J 2017 Temperature-dependent raman responses of the vapor-deposited tin selenide ultrathin flakes J. Phys. Chem. C 121 4674-9

51. Taube A, Łapińska A, Judek J and Zdrojek M 2015 Temperature dependence of Raman shifts in layered ReSe2 and SnSe2 semiconductor nanosheets Appl. Phys. Lett. 107013105

52. Late D J, Shirodkar S N, Waghmare U V, Dravid V P and Rao C N R 2014 Thermal expansion, anharmonicity and temperature-dependent raman spectra of single- and few-layer MoSe2 and WSe2 ChemPhysChem 15 1592-8

53. Yan R, Simpson J R, Bertolazzi S, Brivio J, Watson $M$, Wu X, Kis A, Luo T, Hight Walker A R and Xing H G 2014 Thermal conductivity of monolayer molybdenum disulfide obtained from temperature-dependent raman spectroscopy ACS Nano 8 986-93

54. Yu J, Wang X-H, Feng J, Meng X, Bu X, Li Y, Zhang N and Wang P 2019 Antimonene nanoflakes: extraordinary photoacoustic performance for highcontrast imaging of small volume tumors Adv. Healthc. Mater. 81900378

55. Tao $W$ et al 2018 Two-dimensional antimonene-based photonic nanomedicine for cancer theranostics Adv. Mater. 301802061

56. Duo Y, Huang Y, Liang W, Yuan R, Li Y, Chen T and Zhang H 2019 Ultraeffective cancer therapy with an antimonene-based $x$-ray radiosensitizer Adv. Funct. Mater. 301906010 
57. Niu X, Li Y, Zhang Y, Zhou Z and Wang J 2019 Greatly enhanced photoabsorption and photothermal conversion of antimonene quantum dots through spontaneously partial oxidation ACS Appl. Mater. Interfaces 11 1798793

58. Zhou H et al 2014 High thermal conductivity of suspended few-layer hexagonal boron nitride sheets Nano Res. 7 1232-40

59. Peimyoo N, Shang J, Yang W, Wang Y, Cong C and Yu T 2015 Thermal conductivity determination of suspended mono- and bilayer WS2 by Raman spectroscopy Nano Res. 8 1210-21

60. Judek J, Gertych A P, Świniarski M, Łapińska A, Dużyńska A and Zdrojek M 2015 High accuracy determination of the thermal properties of supported $2 D$ materials Sci. Rep. 512422

61. Zhang T, Qi -Y-Y, Chen X-R and Cai L-C 2016 Predicted low thermal conductivities in antimony films and the role of chemical functionalization Phys. Chem. Chem. Phys. 18 30061-7

62. Ares P, Zamora F and Gomez-Herrero J 2017 Optical identification of few-layer antimonene crystals ACS Photon. 4 600-5

63. Li Q-Y, Xia K, Zhang J, Zhang Y, Li Q, Takahashi K and Zhang X 2017 Measurement of specific heat and thermal conductivity of supported and suspended graphene by a comprehensive Raman optothermal method Nanoscale 9 10784-93

64. Wolff S, Gillen R, Assebban M, Abellán G and Maultzsch J 2020 Twodimensional antimony oxide Phys. Rev. Lett. 124126101

65. Bat-Erdene $M$ et al 2020 Surface oxidized two-dimensional antimonene nanosheets for electrochemical ammonia synthesis under ambient conditions $\mathrm{J}$. Mater. Chem. A 8 4735-9 